\title{
Chitin based polyurethanes using hydroxyl terminated polybutadiene, part III: surface
} characteristics.

\begin{abstract}
Hydroxy terminated polybutadiene (HTPB)-chitin based polyurethanes (PUs) with controlled hydrophobicity were synthesized using HTPB and toluene diisocyanate (TDI). The prepolymer was extended with different mass ratios of chitin and 1,4-butane diol (BDO). The effect of chitin contents in chain extender (CE) proportions on surface properties was studied and investigated. Incorporation of chitin contents into the final PU showed decrease in contact angle value of water drop, water absorption (\%) and swelling behavior. The antibacterial activity of the prepared samples was affected by varying the chitin contents in the chemical composition of the final PU. The results demonstrated that the use of prepared material can be suggested as non-absorbable suture.
\end{abstract}

Keyword: Chitin; Polyurethane; HTPB; Contact angle measurement; Antibacterial activity. 